\title{
Online optimization of storage ring nonlinear beam dynamics
}

\author{
Xiaobiao Huang ${ }^{*}$ and James Safranek \\ SLAC National Accelerator Laboratory, Menlo Park, California 94025, USA
}

(Received 26 February 2015; published 3 August 2015)

\begin{abstract}
We propose to optimize the nonlinear beam dynamics of existing and future storage rings with direct online optimization techniques. This approach may have crucial importance for the implementation of diffraction limited storage rings. In this paper considerations and algorithms for the online optimization approach are discussed. We have applied this approach to experimentally improve the dynamic aperture of the SPEAR3 storage ring with the robust conjugate direction search method and the particle swarm optimization method. The dynamic aperture was improved by more than $5 \mathrm{~mm}$ within a short period of time. Experimental setup and results are presented.
\end{abstract}

DOI: 10.1103/PhysRevSTAB.18.084001

PACS numbers: 41.85.-p, 29.20.db, 29.27.Bd, 29.85.Fj

\section{INTRODUCTION}

In a storage ring accelerator, sextupole magnets are used to cancel the energy dependence of the focusing force of quadrupole magnets (i.e., chromaticity correction). The magnetic forces acting on the beam by the sextupoles are nonlinear with respect to the transverse position of the particles. These periodic nonlinear forces can drive the motion of particles with large oscillation amplitude to nonlinear resonances or even chaotic regime to cause beam loss. Reducing the impact of the nonlinear forces of the sextupoles in order to gain large dynamic aperture and momentum aperture is crucial for the lattice design of a storage ring, and it has become more challenging in recent years as new rings push for lower emittances, which typically require stronger focusing and more lattice periods and in turn more and stronger sextupole magnets. For the new generation of storage ring light sources, that are based on multibend achromat lattice structure and whose emittances reach diffraction limit levels, the nonlinear dynamics challenge is especially severe. Existing storage rings have benefited greatly from robust, beam-based linear optics correction [1]. Future ultralow emittance storage rings may require similar corrections for nonlinear optics.

To attain large dynamic aperture, sextupoles in a storage ring are usually placed with carefully chosen betatron phase advances between them such that the nonlinear kicks on the beam by these magnets partially or fully cancel. Additional sextupole families (harmonic sextupoles) are often used in newer rings to cancel the driving terms of major lowerorder resonances [2]. For both schemes cancellation of the nonlinear kicks relies on accurate betatron phase advance

\footnotetext{
*xiahuang@slac.stanford.edu

Published by the American Physical Society under the terms of the Creative Commons Attribution 3.0 License. Further distribution of this work must maintain attribution to the author $(s)$ and the published article's title, journal citation, and DOI.
}

control between the sextupole magnets. In reality, however, even after successful linear optics correction, it is still common to have residual phase errors on the order of, e.g., $\sigma_{\phi} \sim 0.005-0.01 \mathrm{rad}$. In the case of a large ring with many sextupole magnets, the incomplete cancellation could add up to give large errors to the resonance driving terms (RDTs), which may cause the nonlinear beam dynamics of the real ring to deviate significantly from the design model.

Another source of errors is the differences of the nonlinear elements in the design model and the real ring. These include strength errors of the sextupole magnets, systematic (if not already included in the model) and random multipole errors of magnets and effects of insertion devices. Differences are also introduced by simplifications that are made in the lattice model, such as the use of the "hard-edge" magnet model. Another effect that is commonly ignored in lattice modeling is the cross-talk or interference between the magnetic fields of adjacent magnets. Differences between lattice model and real machine have been observed at the SPEAR3 storage ring [3].

In recent years advanced multiobjective optimization methods have been widely used for the optimization of nonlinear beam dynamics in storage ring lattice design [4-6], which in simulation can significantly improve the dynamic and momentum apertures. However, given the potential differences between the real machine and the lattice model, it is likely that the optimal condition as determined by the model optimization will not give the expected result in the machine. In such a case, experimental correction of the nonlinear optics of the machine may be used to bring the machine to the optimal condition.

Previous beam-based nonlinear dynamics optimization work includes measurement and correction of certain nonlinear dynamics characteristics such as amplitudedependent detuning [7] and RDTs [8,9]. However, these efforts have not yielded definitive evidence that indicates such correction improved the nonlinear dynamics performance measures such as the dynamic and momentum 
apertures. Simulations show that minimizing driving terms does not always lead to bigger dynamic aperture [5]. Therefore, it is possible that correction of the indirect measures will not fully recover the predicted performance.

In this paper, we propose to use beam based measurement to directly optimize the nonlinear dynamics performance with systematic online optimization algorithms. In the following we discuss considerations of the online nonlinear dynamics optimization approach and present experimental results of the application of this approach to the SPEAR3 storage ring.

\section{CONSIDERATIONS OF ONLINE OPTIMIZATION OF NONLINEAR BEAM DYNAMICS}

The nonlinear dynamics performance of a storage ring is ultimately characterized by the dynamic aperture and the momentum aperture, which affect two important operational performance measures, the injection efficiency and the Touschek lifetime, respectively. In fact, the purpose of nonlinear dynamics optimization for a storage ring is to obtain good injection efficiency and long Touschek lifetime. In addition, measuring dynamic aperture and momentum aperture is usually more time consuming than directly measuring injection efficiency and beam lifetime. Therefore the objectives of experimental optimization of ring performance would be injection efficiency and beam lifetime (with lifetime measured under certain fixed, Touschek lifetime dominated machine conditions). Since the evaluation of beam lifetime requires steady beam condition which is not compatible with frequent injection, it is difficult to simultaneously optimize the two objectives for a given lattice. One approach may be to optimize one objective at a time and iterate if necessary.

The optimization variables (i.e., knobs) can be the strengths of sextupole magnets. Since sextupole magnets located in dispersive regions affect the chromaticities, the sextupole knobs should be combined to form independent variables that do not change chromaticities. One way to form such variables is described in the next section. For large rings with an individual power supply for each sextupole magnet, the number of free variables may be reduced by grouping the power supplies according to symmetry or periodicity considerations to form individual knobs. It is also possible to use combinations of quadrupole magnets as optimization variables, if these knobs are set up to meet the linear optics requirements of the storage ring.

Efficiency and robustness are two key requirements for online optimization algorithms. High efficiency means the algorithms need to find the minimum with a small number of evaluations of the objective function(s). Robustness requires the algorithms to be effective under the influence of random noise and occasional outliers in the measurement of the objective function(s). Reference [10] proposed the robust conjugate direction search (RCDS) method for online optimization and demonstrated through simulation and experiments that it is both efficient and robust. The RCDS algorithm is a single-objective method and would stop at a local minimum. However, it is usually a good choice for online nonlinear dynamics optimization, because the injection efficiency and lifetime objectives normally need to be optimized separately and in most cases the global minimum is not far from the starting point, which is typically set according to an optimized model. Convergence is fast in the vicinity of the minimum for conjugate direction search methods such as RCDS.

The RCDS may also be used in the case when the starting point is far from the global minimum (e.g., for the linear coupling correction problem as demonstrated in Ref. [10]). However, if a more thorough search of the parameter space is desired in order to gain more assurance that the global minimum is reached, stochastic optimization algorithms such as genetic algorithms [4,5,11,12] and particle swarm optimization (PSO) algorithms [6,13,14] can be used. Studies have indicated the particle swarm method is a few times more efficient than a widely used genetic algorithm because solutions evaluated by the former are considerably more diverse than that of the latter. In addition, Ref. [10] showed that, for genetic algorithms, solutions biased by random errors tend to enter the next generation and therefore hamper the convergence to the true global minimum. For these reasons the particle swarm method may be the preferred stochastic optimization algorithm for online applications.

In the following we will discuss the online nonlinear dynamics optimization for the SPEAR3 storage ring with the RCDS and PSO methods, which may serve as an illustration of the above considerations.

\section{ONLINE OPTIMIZATION OF DYNAMIC APERTURE FOR SPEAR3}

SPEAR3 is a third generation storage ring light source. It has a total of 72 sextupoles, 36 focusing sextupoles (SF) and 36 defocusing sextupoles (SD), with two SFs and two SDs in each of its 18 cells (14 standard cells and 4 matching cells). The 72 sextupoles used to be powered by 4 power supplies for chromaticity correction. During the 2014 summer shutdown, 6 additional sextupole power supplies were added, resulting in a total of 10 sextupole families. Each family consists of several sextupoles of the same type (SF or SD). These extra sextupole power supplies enabled the online nonlinear dynamics optimization to be discussed below.

The response matrix of chromaticities with respect to the 10 sextupole families, $\mathbf{R}_{\xi}$, is calculated using the lattice model. To keep chromaticities fixed during optimization, it is sufficient and necessary that any changes to the sextupoles be in the null space of $\mathbf{R}_{\xi}$, which is a 2 by 10 matrix. With singular value decomposition (SVD), $\mathbf{R}_{\xi}=\mathbf{U S V}^{T}$, the null space is simply the subspace spanned by the 8 
vectors in $\mathbf{V}$ which correspond to zero singular values. These 8 vectors represent a basis of the null space of the chromaticity response matrix, so they were used as the free knobs in our nonlinear dynamics optimization.

When using the RCDS method, it is recommended to find an initial conjugate direction set in order to improve searching efficiency [10]. Such a direction set could be obtained with the Jacobian matrix of some selected nonlinear RDTs or the distortion functions as proposed by Collins [15] with respect to the 8 free knobs. But since it is unclear if the direction set will be effective, and for simplicity, we simply used the 8 unit vectors as the initial directions.

The objective function is the injection efficiency. It is evaluated as the increase of stored beam current divided by the average intensity of the injector beam for an interval of 10 seconds. Noise in the objective function comes mainly from the uncertainty of the injected beam intensity measurement. The noise sigma of injection efficiency is about $3 \%$.

We have applied online dynamic aperture optimization for the SPEAR3 operational lattice with both the RCDS and the PSO algorithms. Since this lattice normally has good injection efficiency, we intentionally scaled down the injection kicker bump, which effectively increased the dynamic aperture requirement for capturing the injected beam. Figure 1 top plot shows the history of the objective function of all evaluated solutions during an RCDS optimization. In this experiment, the kicker bump was first reduced to $85 \%$ of the original full bump, and then was further reduced to $77 \%$ as injection efficiency was
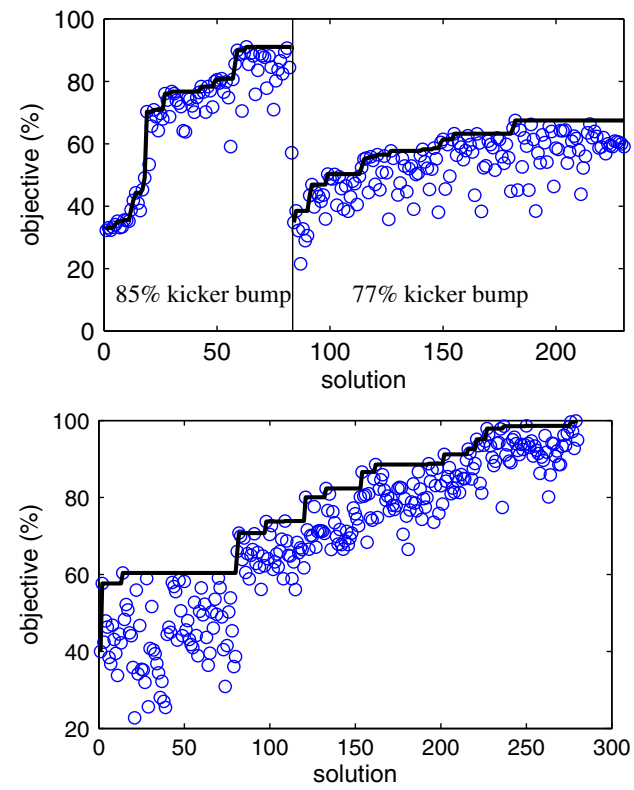

FIG. 1. History of objective function values of all evaluated solutions during the RCDS optimization (top) and the PSO optimization (bottom). improved. This RCDS run was terminated after three iterations of the direction set scans were completed.

The PSO method was applied to the same problem after a sizable improvement $(\sim 2.5 \mathrm{~mm})$ of dynamic aperture was made with a preliminary RCDS run. The population of solutions for the PSO run was 40. The initial population was centered around the solution (which was included) found by the preliminary RCDS run. Deviation of each variable value of the initial solutions was drawn from a random uniform distribution, whose size is $\pm 10 \%$ of its range. The kicker bump was reduced so that the initial injection efficiency was about $40 \%$. The algorithm was run for 7 generations and was stopped when the injection efficiency reached $100 \%$. The history of the objective function of the PSO optimization was shown in Fig. 1 bottom plot.

The sextupole settings of the RCDS and PSO solutions are compared to the original values in Fig. 2. The sextupole strengths in the standard cells for the optimized solutions are changed substantially. The solutions found by PSO and RCDS are very similar. Chromaticities were measured and it was found that the horizontal and vertical chromaticities of all optimized solutions were equal to the original values of $\xi_{x}=\xi_{y}=+3$.

Dynamic aperture measurement was done to characterize the optimized solutions. In this measurement a short train of bunches of stored beam was kicked by one of the injection kickers with increasing kick strength until all beam was lost. The kicker was fired 10 times at each kick strength. Figure 3 top plot shows the fractional beam current change vs kicker voltage, where an arbitrary line at 50\% of fractional loss indicates the kick strengths that drive the beam to the edge of the dynamic aperture for the three cases. The kick angles are used in particle tracking with the corresponding lattice models for 200 turns. The resulted phase space plots for the two optimized solutions are too close to discern. In Fig. 3 bottom plot we only show a comparison of the PSO solution ("optimized") and the original sextupole setting. If the half size of the stored beam $\left(2 \sigma_{x}=0.6 \mathrm{~mm}\right)$ is included, the dynamic aperture for the

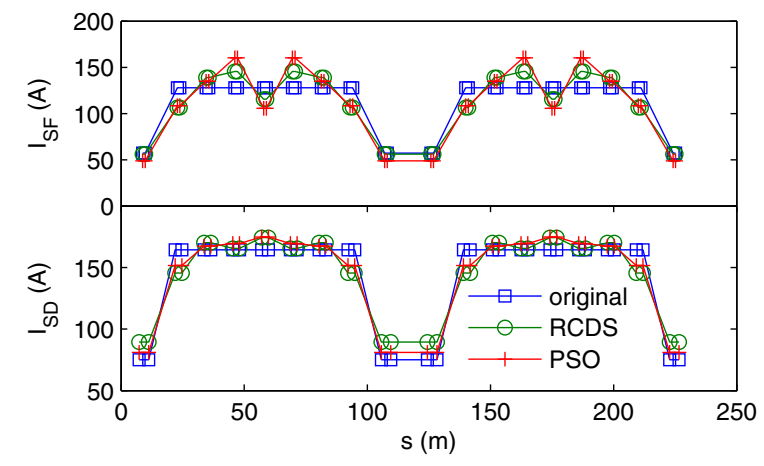

FIG. 2. Comparison of sextupole settings for the original and optimized solutions. 

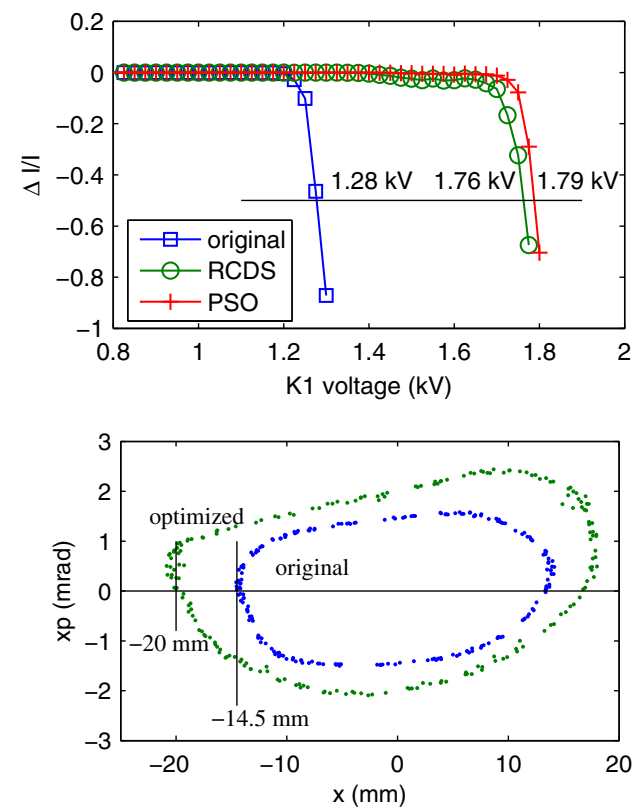

FIG. 3. Comparison of measured dynamic aperture for the original and optimized solutions.

original and optimized solutions are 15.1 and $20.6 \mathrm{~mm}$, respectively.

The dynamic aperture gain for the optimized solutions was further verified with the measurement of injection efficiency vs the injection kicker bump. The strengths of all three injection kickers were scaled to vary the size of the kicker bump and the injection efficiency was checked at each step. The results for the original and optimized sextupole settings are shown in Fig. 4. The kicker bumps for both solutions were closed (i.e., there was no residual oscillation for the stored beam.) for the measurements. The measurements confirmed that the dynamic aperture was increased by more than $5 \mathrm{~mm}$ after the online optimization.

Beam lifetime under operation conditions $(500 \mathrm{~mA}$ in 280 bunches, with rf voltage of $2.85 \mathrm{MV}$ ) was found to be about $6.8 \mathrm{hrs}$ for the optimized solution, compared to $7.8 \mathrm{hrs}$ for the original sextupole setting. Subsequent

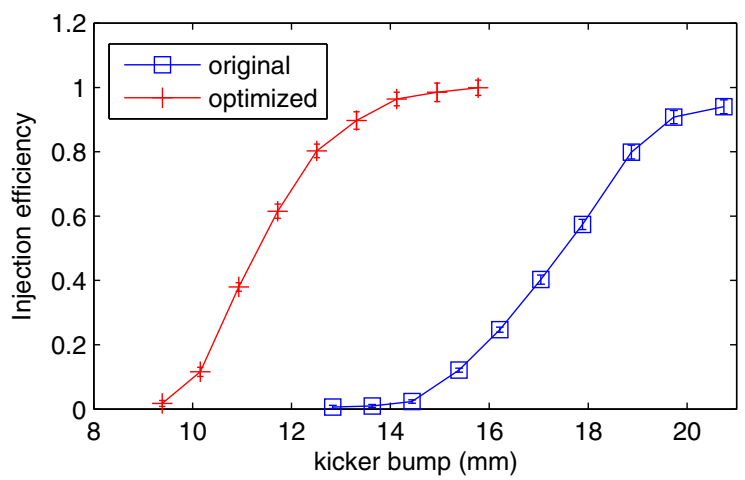

FIG. 4. Injection efficiency vs kicker bump for the original and optimized solutions. measurements verified that this lifetime reduction was simply a result of smaller vertical beam size for the optimized solution, and not a momentum aperture problem. To check for possible reduction of momentum aperture for the optimized solution, we measured the beam lifetime under the Touschek lifetime dominated regime while reducing the rf voltage. As shown in Fig. 5, for the optimized solution the lifetime went down almost monotonically with a decreasing if voltage when it was below 3.1 MV, which indicates that the momentum aperture is determined by the rf bucket height rather than a reduced off-momentum dynamic aperture [16]. In addition, the lifetime of the optimized solution is proportionally lower than the original solution, even for very low rf voltages where the momentum aperture is certainly determined by the rf bucket height. Therefore, the reduced lifetime at $500 \mathrm{~mA}$ for the optimized solution is not due to a reduced local momentum aperture.

To verify that the cause of the lifetime difference was change to the vertical emittance due to the sextupole changes, in a later shift, we set the vertical to horizontal emittance ratio to equal values for the two solutions and compared the beam lifetime. The emittance ratio was $0.088 \%$ (optimized solution) and $0.087 \%$ (original solution), respectively, as measured by the orbit response matrix fitting method, LOCO [1]. The vertical beam size measured by the pinhole camera for the two sextupole solutions were $17.8 \pm 0.5 \mu \mathrm{m}$ (optimized) and $18.2 \pm 0.5 \mu \mathrm{m}$ (original), respectively. The corresponding lifetime at $497 \mathrm{~mA}$ for the two solutions were nearly identical: $7.68 \mathrm{hr}$ (optimized) and $7.66 \mathrm{hr}$ (original).

The dynamic apertures for the original and optimized sextupole settings have been evaluated with particle tracking simulation. The simulation includes realistic vertical physical aperture, effects of insertion devices and systematic magnetic multipole errors. Random linear and nonlinear errors are also added with 20 random seeds. The linear optics error was corrected to $1 \% \mathrm{rms}$ beta beating and $0.2 \%$ linear coupling, close to realistic values.

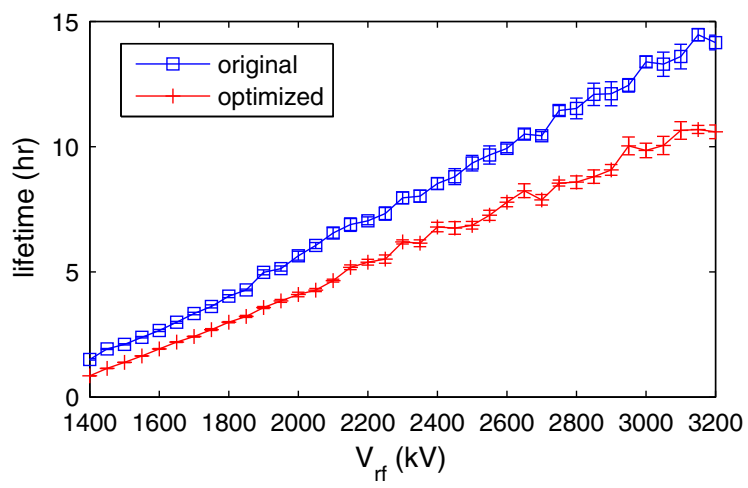

FIG. 5. Lifetime vs rf gap voltage for the original and optimized solutions. Initial current was $100 \mathrm{~mA}$ in 80 bunches for both cases, which was Touschek lifetime dominated. 


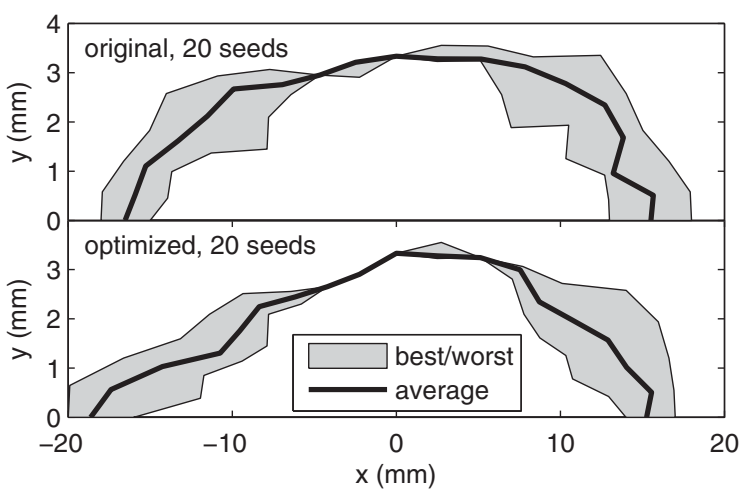

FIG. 6. Dynamic aperture obtained by particle tracking for the original (top) and optimized (bottom) solutions.

Results of simulated dynamic aperture for the original and the optimized solutions are shown in Fig. 6. Interestingly, the dynamic aperture determined by tracking for the optimized solution indeed has a substantial gain on the negative side of the horizontal midplane where the injected beam comes in. But there is a dent on the vertical direction near $x=-10 \mathrm{~mm}$. If this dent is real for the actual machine, it indicates the experimentally optimized solution found a better balance between the horizontal and vertical dynamic aperture requirements. Optimizing the dynamic aperture and momentum aperture using the model in simulation for a given random error seed with the multiobjective particle swarm method yielded solutions with dynamic aperture that extends to $-20 \mathrm{~mm}$ with no vertical dent. Therefore it is also possible that the optimized real machine has no such a dent in the dynamic aperture and the discrepancy may come from the differences between the lattice model and the machine $[3,17]$.

As we improve the agreement between the lattice model and the real machine with more accurate models for accelerator elements and with beam-based nonlinear optics calibration techniques, our ability to predict and control the nonlinear dynamics behavior of the machine will increase. In this study we showed that the online optimization method can be very useful before a perfect agreement between the lattice model and the machine is reached.

\section{CONCLUSION}

We propose to optimize the nonlinear dynamics of storage rings online directly with advanced optimization algorithms. Because of the intrinsic differences between real machines and their corresponding lattice models, ideal performance of optimized models may not be directly achieved on the real machine. The online approach could bridge the gap between the optimized model and the actual machine to achieve the optimal performance.

We have demonstrated the power of the online methods with dynamic aperture optimization for the SPEAR3 storage ring. In the experiments the dynamic aperture of SPEAR3 was improved from $15.1 \mathrm{~mm}$ to $20.6 \mathrm{~mm}$, while the momentum aperture was not negatively affected.

\section{ACKNOWLEDGMENTS}

The study is supported by DOE Contract No. DE-AC02$76 \mathrm{SF} 00515$.

[1] J. Safranek, Nucl. Instrum. Methods Phys. Res., Sect. A 388, 27 (1997).

[2] J. Bengtsson, SLS Report No. 9/97, 1997 (unpublished).

[3] J. Safranek, X. Huang, J. Corbett, J. Sebek, and A. Terebilo, in Proceedings of the 23rd Particle Accelerator Conference, Vancouver, Canada, 2009 (IEEE, Piscataway, NJ, 2009), p. 3928.

[4] M. Borland, V. Sajaev, L. Emery, and A. Xiao, in Proceedings of the 23rd Particle Accelerator Conference, Vancouver, Canada, 2009 (IEEE, Piscataway, NJ, 2009), p. 3851.

[5] L. Yang, Y. Li, W. Guo, and S. Krinsky, Phys. Rev. ST Accel. Beams 14, 054001 (2011).

[6] X. Huang and J. Safranek, Nucl. Instrum. Methods Phys. Res., Sect. A 757, 48 (2014).

[7] R. Bartolini, I. P. S. Martin, G. Rehm, and F. Schmidt, Phys. Rev. ST Accel. Beams 14, 054003 (2011).

[8] R. Bartolini, I. P. S. Martin, J. H. Rowland, P. Kuske, and F. Schmidt, Phys. Rev. ST Accel. Beams 11, 104002 (2008).

[9] A. Franchi, L. Farvacque, F. Ewald, G. Le Bec, and K. B. Scheidt, Phys. Rev. ST Accel. Beams 17, 074001 (2014).

[10] X. Huang, J. Corbett, J. Safranek, and J. Wu, Nucl. Instrum. Methods Phys. Res., Sect. A 726, 77 (2013).

[11] I. V. Bazarov and C. K. Sinclair, Phys. Rev. ST Accel. Beams 8, 034202 (2005).

[12] K. Tian, J. Safranek, and Y. Yan, Phys. Rev. ST Accel. Beams 17, 020703 (2014).

[13] Y. Wang, M. Borland, and V. Sajaev, in Proceedings of the 24th Particle Accelerator Conference, PAC-2011, New York, 2011 (IEEE, New York, 2011), p. 787.

[14] X. Pang and L. Rybarcyk, Nucl. Instrum. Methods Phys. Res., Sect. A 741, 124 (2014).

[15] T. Collins, Fermilab Report No. 84/114, 1984.

[16] C. Steier, D. Robin, L. Nadolski, W. Decking, Y. Wu, and J. Laskar, Phys. Rev. E 65, 056506 (2002).

[17] X. Huang, J. Safranek, and D. Dell'Orco, in Proceedings of the International Particle Accelerator Conference, Kyoto, Japan (ICR, Kyoto, 2010), p. 4627. 